\title{
UNA VASIJA-SONAJA MAYA DE DOBLE FONDO *
}

\author{
Por Hasso von Winning \\ Southwest Museum, \\ Los Angeles, California
}

Presentamos en la fig. 1 a-c una vasija semiglobular de doble fondo, modelada y grabada en su exterior, que merece la atención por su extraordinaria técnica de elaboración, su simbolismo y perfecto estado de conservación. En realidad se trata de dos vasijas: una de lados ligeramente convergentes y otra de lados más cortos cuyos bordes están fundidos a la primera con tal perfección, que da la impresión de tratarse de un solo cajete. El espacio entre ambos fondos contiene algún material granuloso fino el que, al agitarse, produce un sonido que sugiere el ruido de la lluvia.

La vasija es de color café negruzco, muy bien pulida, con un diámetro de $16.8 \mathrm{cms}$., $13 \mathrm{cms}$. de altura y una distancia máxima entre los fondos de $1.8 \mathrm{cms}$. La decoración grabada e incisa fue hecha antes de la cochura, quedando algunos restos de cinabrio en las porciones grabadas. Un gran número de perforaciones están distribuidas de tal manera en la parte grabada que no afectan los motivos decorativos. La vasija proviene del sureste de México, sin poder precisarse el lugar, y se encuentra actualmente en "The Dumbarton Oaks Collections of Pre-Columbian Art”, de Washington (Colección Bliss).

\section{Generalidades}

Sobre la distribución de este tipo de vasijas, que es bastante raro, Berlin observa que por lo general son de barro gris fino y que parecen estar limitadas a los estados de Tabasco, Cam-

* Agradezco las observaciones que después de haber leído el manuscrito tuvieron a bien hacer los profesores Dr. Franz Termer y Dr. Günter Zimmermann (ambos de la Universidad de Hamburgo) y el Dr. Joaquín Weihmann (México, D. F.). 
peche y Yucatán. Se distinguen dos centros de mayor ocurrencia, uno en el noroeste de la Península y otro en la región de Jonuta, sobre el Usumacinta, pero no ha sido posible determinar cuál de las dos regiones fue el centro de manufactura. El citado investigador piensa que las vasijas de doble fondo pertenecen al final del Clásico Tardío (Berlin, 1956, pp. 118119). Un ejemplar con pequeños soportes, de Jonuta, presenta decoración incisa representando un mono, con el fondo del dibujo marcado por "dentate stamping", un método decorativo sumamente raro en Mesoamérica (Shook, 1957, pp. 285-287). Se distinguen, además, tres tipos de vasijas-sonaja con base de pedestal y de barro anaranjado fino, cuya distribución es más amplia en el área maya que el tipo de vasija que aquí describimos.

\section{Decoración}

A lo largo del borde hay una serie de impresiones hechas con el pulgar (fig. 1-a). Debajo de ellas se extiende una faja con once motivos ovalados idénticos, cada uno con dos volutas laterales. Los cartuchos están divididos en cuatro secciones, dos de las cuales, o sea la superior izquierda y la inferior derecha están cuadriculadas (fig. 3). Este diseño se asemeja al Glifo 1305 de Zimmermann (fig. 2-a) cuyos elementos forman parte del Glifo 593 de Thompson (fig. 2-b) (Zimmermann, 1956, p. 81; Thompson, 1962, p. 220). Las volutas corresponden al Afijo Th 129.

Basándonos en estas comparaciones pensamos que ciertos elementos decorativos en la vasija fueron derivados de glifos. La presencia de estos glifos en pasajes del Códice Madrid aclara la función y el simbolismo de la vasija.

Por ejemplo, el Glifo 1305 (Zimmermann) lo encontramos en Madrid 67, asociado, aparentemente, con un dios sentado que lleva una sonaja en la mano, lo que sugiere una relación con la sonaja incorporada en la vasija. Además, el Glifo 1305 con el coeficiente 9 está asociado con el dios de la lluvia (Zimmermann, 1956, p. 81). Volveremos a este glifo al tratar de la decoración en el cuerpo de la vasija.

Entre el borde y el cuerpo globular hay una franja lisa con cuatro prominencias circulares puntiagudas apuntando a las cuatro direcciones de la brújula. 

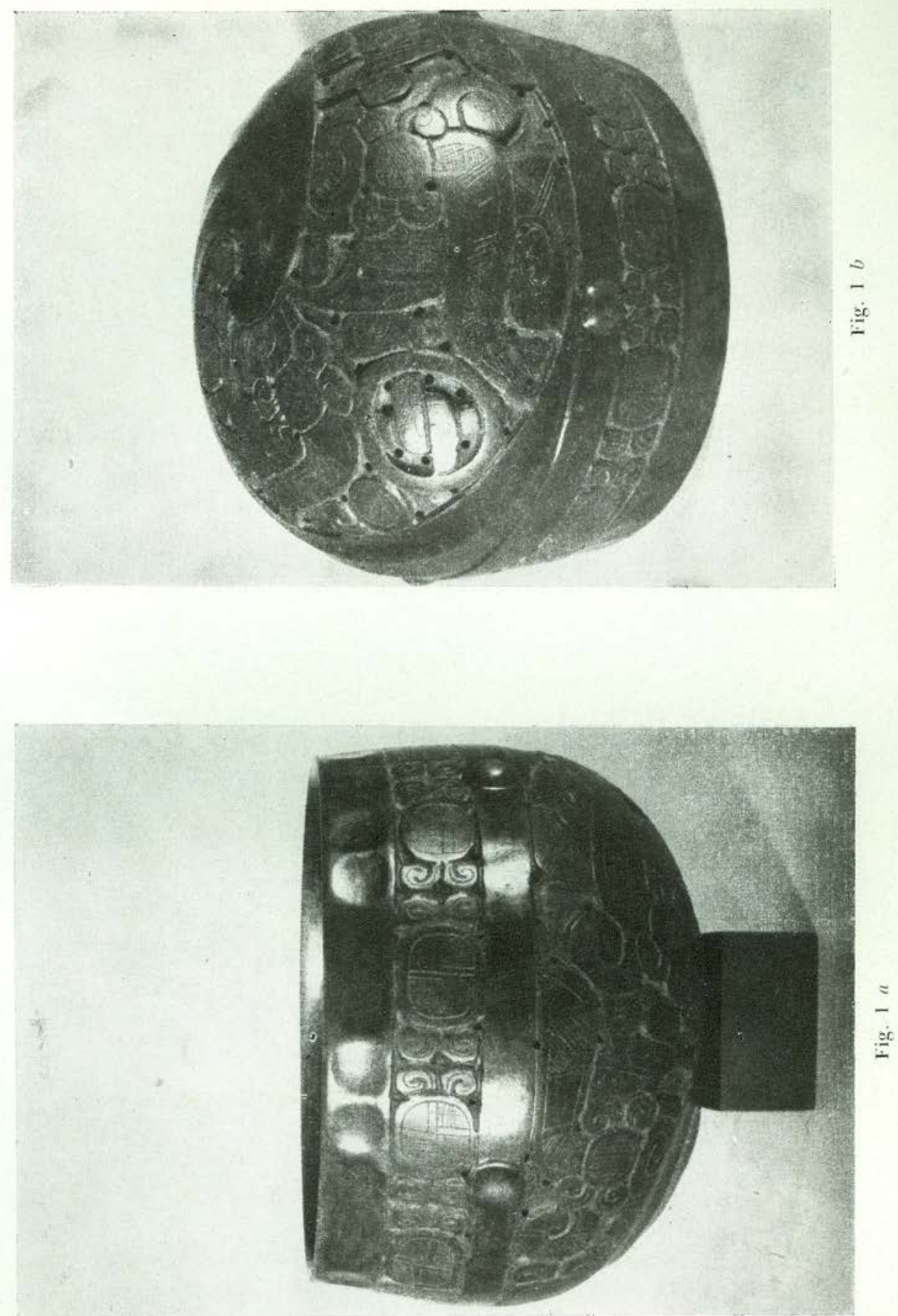

Estudios de Cultura Maya. Vol. VI, 1967

Facultad de Filosofía y Letras, UNAM

http://www.iifilologicas.unam.mx/estculmaya/ 


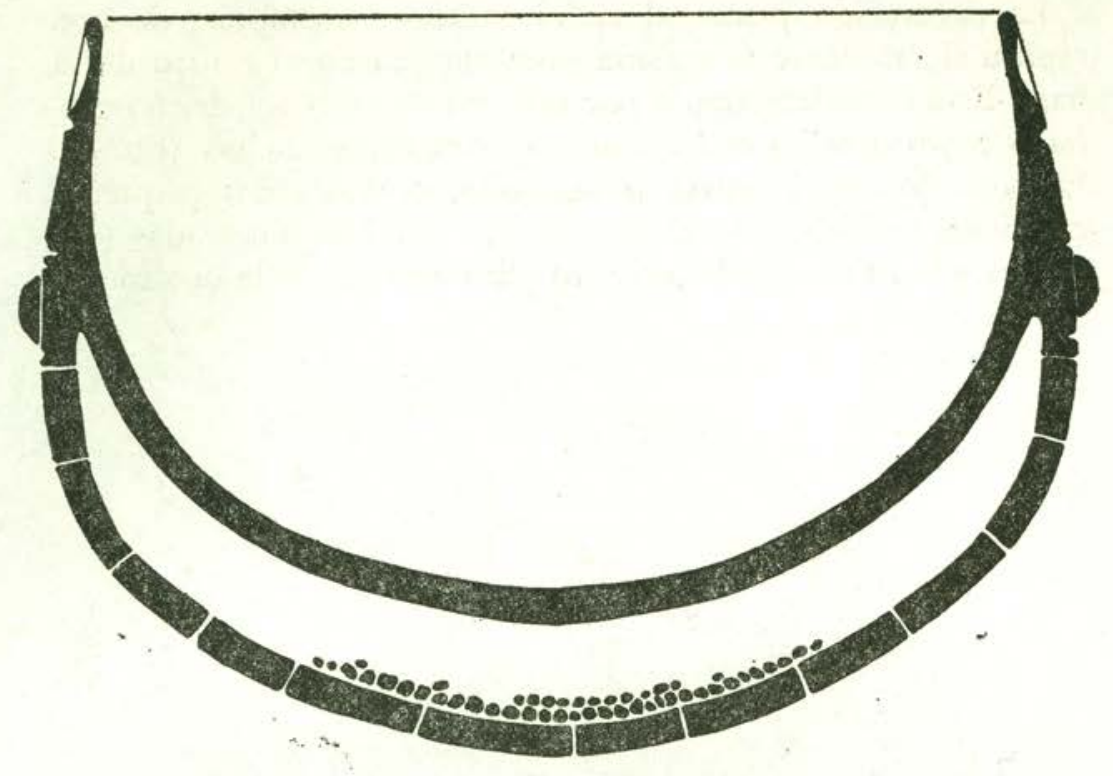

Fig. $1 c$
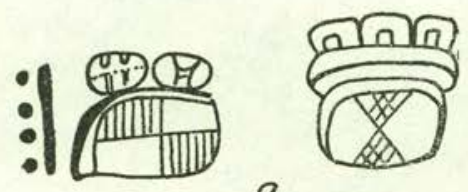

a
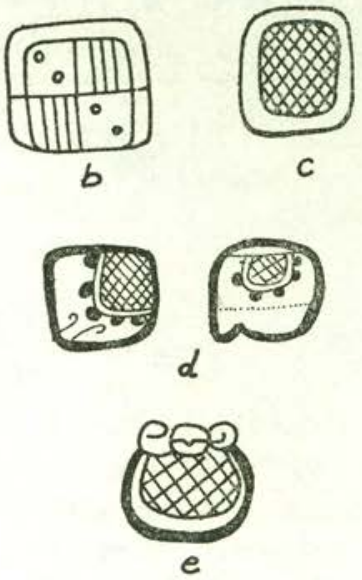

Fig. 2 
La decoración principal en el cuerpo se compone de una espiral ligeramente acanalada que empieza en el centro de la base. Está complementada por otra espiral con un diseño grabado cuyos detalles están indicados con líneas incisas (fig. 3) . Empezando con la parte puntiaguda, notamos dos pequeños cartuchos ovalados, el segundo de ellos con líneas cruzadas (fig. 3: 1, a y b). En seguida hay otro glifo igual al de la decoración

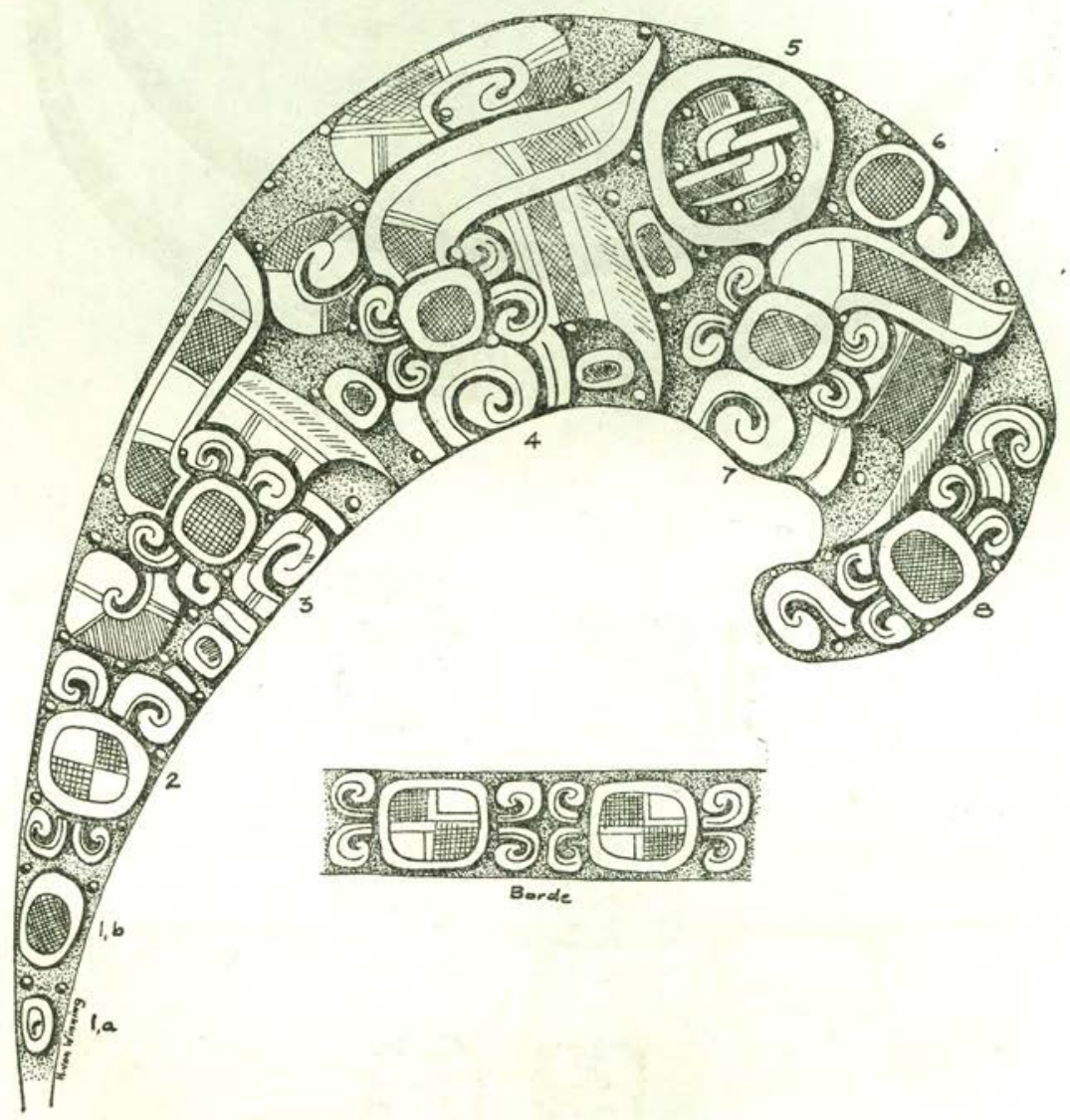

Fig. 3

del borde superior (fig. 3: 2). Siguen tres motivos casi idénticos que se caracterizan por grandes volutas alrededor del glifo en el borde, sin embargo los tres cartuchos centrales no están seccionados en cuatro partes, sino rellenados completamente con líneas cruzadas (fig. 3: 3, 4 y 7). El siguiente motivo (fig. 
3: 8) es más sencillo por falta de espacio, y por lo tanto se trata de una forma abreviada de los tres anteriores.

¿Qué significan pues, los tres grandes motivos similares con el otro que está abreviado? Pensamos que se trata de una estilización de cabezas de serpientes, por las siguientes razones:

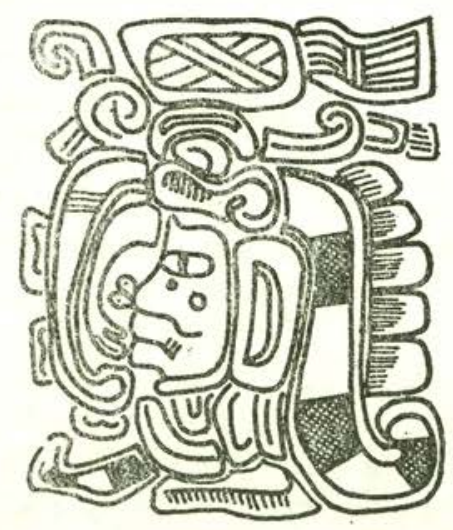

Fig. 4

El elemento central en cada ejemplar es el Glifo Th 586a (hatched dot) que ocurre tanto en los códices como en murales y en cerámica. Va acompañado de volutas (Afijo Th 129). Debajo de este motivo central hay una voluta grande (que suponemos representa el ojo) y encima un elemento longitudinal con dos secciones y con líneas marginales. Estos detalles se ven claramente en la mandíbula abierta de la serpiente en

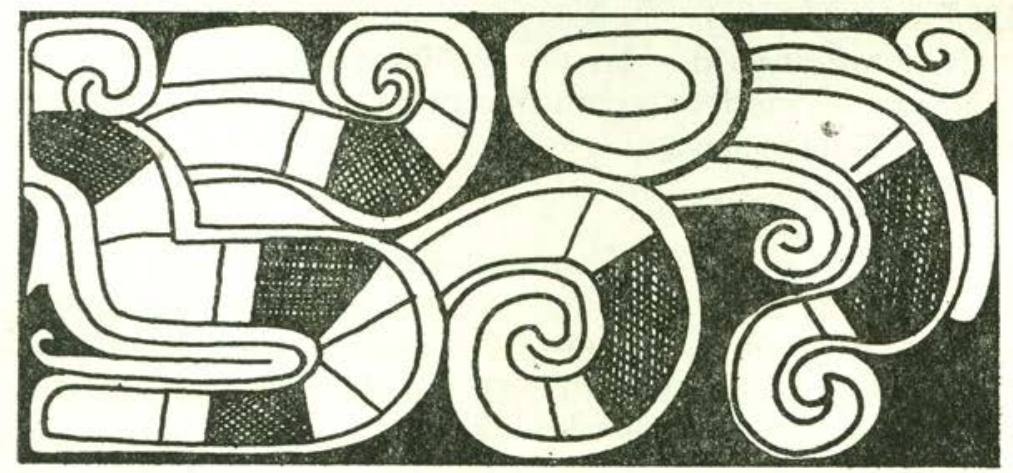

Fig. 5 
un vaso del Periodo Clásico Temprano de Kaminaljuyú (fig. 4) y en una vasija incisa de Patal, Municipio Tactic, Alta Verapaz (fig. 5). Rayas cruzadas también forman las manchas en los cuerpos de las serpientes que descienden del cielo en medio de corrientes de agua (indicando lluvia) en Madrid $12, \mathrm{~b}-15$, b y tales manchas figuran también en el signo del día Chicchan (fig. 2, d). Por lo tanto los cuatro motivos (fig. 3: $3,4,7$, y 8 ) probablemente representan los cuatro Chicchanes, las grandes serpientes que residen en el cielo en los cuatro puntos cardinales y que distribuyen la lluvia (Thompson, 1960 , p. 75$)$.

Finalmente, entre los motivos serpentinos vemos un gran ornamento circular con bandas entrelazadas (fig. 3: 5) que forma parte del signo del primer mes, Pop, pero que es también un motivo decorativo frecuente en el arte maya (Glifo Th 615, Thompson, 1962, p. 237) ; nótese que las bandas cruzadas también aparecen encima de la cabeza de serpiente en la figura 4. Además, para evitar espacios vacíos, algunas variantes del signo "hatched dot" están distribuidos en el diseño (fig. 3: 6).

Una decoración en general muy parecida, con motivos serpentinos y con el emblema de las bandas entrelazadas, se nota en una vasija de la región de Chamelco (Alta Verapaz) ilustrada por Dieseldorff. Aparentemente se trata de un cajete sin doble fondo, grabado e inciso, cuya decoración en el borde consiste de impresiones rectangulares angostas. El gran emblema de bandas entrelazadas figura en la base y se asemeja al símbolo Nahua que, siglos más tarde, significaba oro cuando ostentaba además cuatro círculos o discos (fig. 6. Dieseldorff, 1933, figs. 83 y 85 ).

Es significativo que las tres vasijas que mencionamos para comparar los motivos serpentinos provienen todas de las tierras altas de Guatemala.

\section{Conclusiones}

¿Qué, pues, es la función y el significado de la decoración en la vasija? Todos los componentes tienden a relacionarla con ceremonias a los dioses de la lluvia:

1) Ya mencionamos la sugestión del sonido de la lluvia cau- 
sado por las piedrecillas entre el doble fondo que forma la sonaja.

2) El arreglo de la decoración en forma de espiral tiene significado acuático, pues las espirales simbolizan el agua tanto en el arte maya como en los códices mexicanos (Thompson, 1962, p. 278).

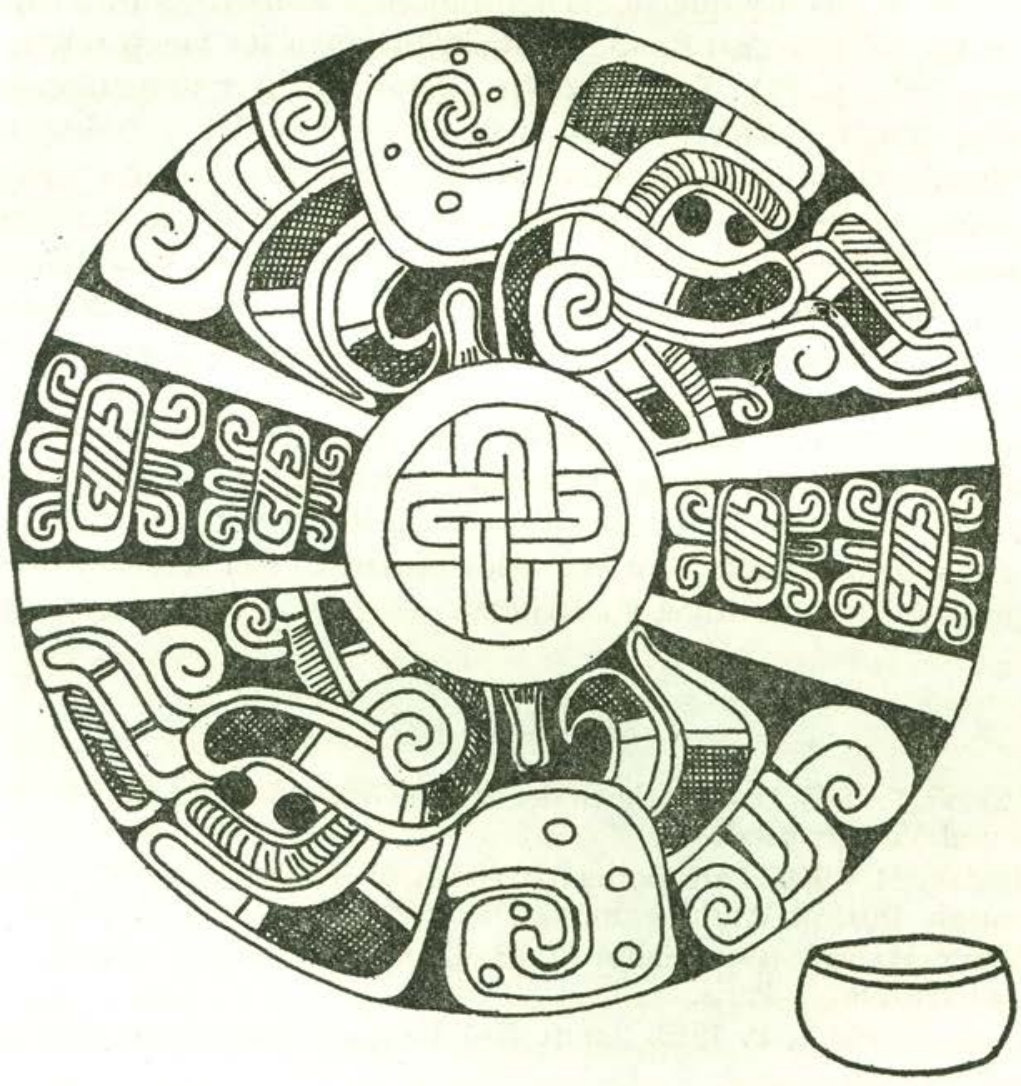

Fig. 6

3) El Glifo 1305 (Zimmermann), que aparece en el borde superior y una vez en el diseño central (fig. 3: 2) está relacionado estrechamente con el Glifo 593 de Thompson. Este último lo observó en las vasijas de las cuales los dioses narigudos vierten el agua, en la estela P de Quirigua. Piensa, por lo tanto, que posiblemente las vasijas decoradas con este glifo 
fueron usadas por los dioses de la lluvia para guardar en ellas el preciado líquido (Thompson, 1962, p. 22, y 1960, fig. 44: 4) .

4) Existe cierta analogía entre el simbolismo expresado en la vasija de doble fondo y la escena en Madrid 74b, que representa cuatro personajes con cuatro grandes ollas. Cada individuo tiene la mano sumergida en su olla para sacar algo (jagua?). Anders propone que se trata de las cuatro ollas que emiten la lluvia y que se encuentran en los cuatro puntos cardinales del universo donde también originan los vientos (Anders, 1963, p. 87). Encima de los personajes hay numerales y glifos que se refieren a las cuatro secciones del tonalámatl. Además, el Glifo 586a (hatched dot) está incluido tres veces en esta escena del Madrid $74 \mathrm{~b}$, con un superfijo de volutas muy parecido a las vírgulas en el motivo central en la vasija.

5) Las cuatro protuberancias en el borde parecen indicar, por su posición, una relación con las cuatro direcciones de la brújula.

En resumen podemos concluir que la vasija de doble fondo probablemente proviene del noroeste del área maya; que haya servido en ceremonias a los dioses de la lluvia y que su decoración probablemente representa los cuatro Chicchanes mayores que dispensan la lluvia y el trueno.

\section{REFERENCIAS}

Anders, F. 1963. Das Pantheon der Maya. Graz, Akademische Druckund Verlagsanstalt.

BerLin, H. 1956. Late pottery horizons of Tabasco, Mexico. Carnegie Inst. of Washington, Contribution № 59, pp. 95-153.

Codex Madrid (Cortesiano). 1883. Codex Cortesianus. Manuscrit hieratique.... Paris.

DieseldorfF, E. P. 1933. Kunst und Religion der Mayavölker III. Hamburg.

Kidder, Alfred II y C. Samayoa Chinchilla. 1959. The art of the ancient Maya. New York.

Sноок, E. M. 1957. Dentate and dentate rocker stamping from Tabasco. American Antiquity, 22:3, pp. 285-287.

Thompson, J. E. S. 1960. Maya hieroglyphic writing. Norman.

—. 1962. A catalog of Maya hieroglyphs. Norman.

ZimmermanN, G. 1956. Die Hieroglyphen der Maya-Handschriften. Universitàt Hamburg, Abh. a. d. Gebiet d. Auslandskunde, Bd. 62. 